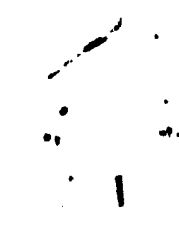

Rerond ty 60 ?

JAN 281991

SAND $-91-0139 \mathrm{C}$

DE9 1006778

\title{
DESIGN CONSIDERATIONS FOR MULTI COMPONENT MOLECULAR-POLYMERIC NONLINEAR OPTICAL MATERIALS
}

\author{
K.D. Singer \\ Case Western Reserve University \\ Department of Physics, Cleveland, Ohio $\$ 1106$ \\ M.G. Kuzyk \\ Washington State University \\ Department of Physics, Pullman, Washington 99164-2814 \\ T. Fang and W.R. Holland \\ ATET Bell Laboratories \\ P.O. Box 900, Princeton, NJ 08540 \\ P.A. Cahill \\ Sandia National Laboratories \\ Division 1811, Albuquerque, NM 87185-5800
}

\begin{abstract}
We review our work on multi component polymeric nonlinear optical materials. These materials consist of nonlinear optical molecules incorporated in a polymeric host. A crosslinked triazine polymer incorporating a dicyanovinyl terminated azo dye was found to be relatively stable at $85^{\circ} \mathrm{C}$ and possess an electro-optic coefficient of $11 \mathrm{pm} / \mathrm{V}$. We have also observed the zero dispersion condition in a new anomalous dispersion dye for phase matched second harmonic generation, and expect efficient conversion to the blue. A squarylium dye, ISQ, has been found to possess a large third order nonlinearity, and may display two-level behavior.

\section{Introduction}

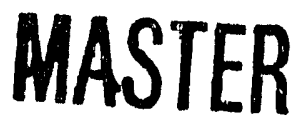

Nonlinear optical materials will provide the functionality required for photonic processing elements. Optical nonlinearity, however, is but one of the required material properties in an optical processing system. The architecture of the system defines, not only the processing element, but also many other requirements of the constituent materials and devices. Examples of other required properties are optical losses, which must satisfy the system power budget, and the material compatibility with the interconnection processes required to assemble the system.


Because of these multiple constraints, single component materials often are not apprc riate. Therefore, much of the recent research effort devoted to the development of appriopriate nonlinear optical materials, both organic and inorganic, has focused on multi component materials. [1] For organic materials, this research has largely centered on guest-host or functionalized polymers, multi component Langmuir-Blodgett films, and mixed crystals. [2] One very promising line of work is directed at electro-optic polymers due to the potential of electro-optic elements in switching systems. Polymeric materials show promise in meeting the many requirements for the switching fabric, such as switching voltage, optical loss, and dielectric properties. [3] A major unresolved issue relating to polymer electro-optic materials is their thermal and temporal stability.

The concept of separate property optimization through the selection and interplay of the components in multi component materials bas been applied by us and others in a variety of ways to polymer nonlinear optical materials. [4] In this paper, we will review our own work in multi component materials as it has been applied to electro-optic materials, second harmonic generation, the control of the nonlinear optical tensor through stress, and to third-order nonlinear optical materials.

\section{Electro-optic Materials}

Substantial effort has gone into optimizing multi component polymeric electro-optic materials since it was first recognized that doped and functionalized poled polymeric materials possess many of desirable properties for electro-optic applications. Polymer glasses possess favorable optical loss properties, and can be fabricated into guided-wave structures using a variety of processing techniques. In addition, the low dielectric constants found in polymer glasses allow electro-optic devices fabricated from them to be operated at high speed. [5] These desirable system properties can be combined with the large optical nonlinearities of organic molecules to produce electro-optic materials. Since the linear electro-optic effect arises from the second-order nonlinear optical susceptibility, materials exhibiting this effect cannot possess a center of symmetry. To meet this requirement, polymers incorporating nonlinear optical molecules must be processed with an electric field in order to orient the molecules in the polymer. [6] This process is depicted in Figure 1 for a dipolar molecule doped into a thermoplastic polymer. The resulting nonlinear susceptibility is given by,

$$
\chi^{(2)}=N \frac{\beta \mu E_{p}}{5 k T}
$$

where $N$ is the number density of nonlinear optical molecules, $\beta$ the molecular noulinear susceptibility, $\mu$ the ground state dipole moment, and $E_{p}$ the poling field. Analysis of Equation 1 indicates that rather large molecular susceptibilities are required to produce useful $\chi^{(2)}$ materials. This is most easily accomplished with visible wavelength absorbing dyes, thus making electro-optic applications, where only one near infrared wavelength is required, most promising.

A number of appropriate material systems have been reported, most based on donor and acceptor substituted extended aromatic systems which have been incorporated in various polymer glasses. An early example of this is a system composed of disperse red 1 , an azo dye, dissolved in polymethylmethacrylate (PMMA). The measured second harmonic generation coefficient, $d_{33}$, of $6 \times 10^{-9}$ esu was found to be in agreement with the oriented gas model 


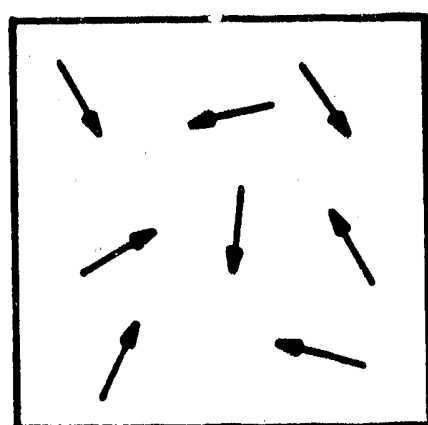

glassy, random orientotion
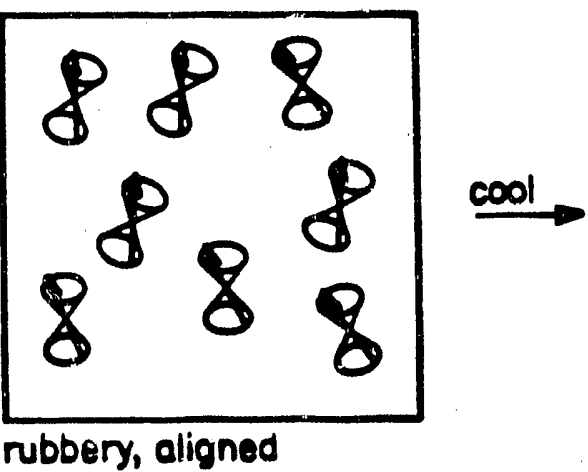
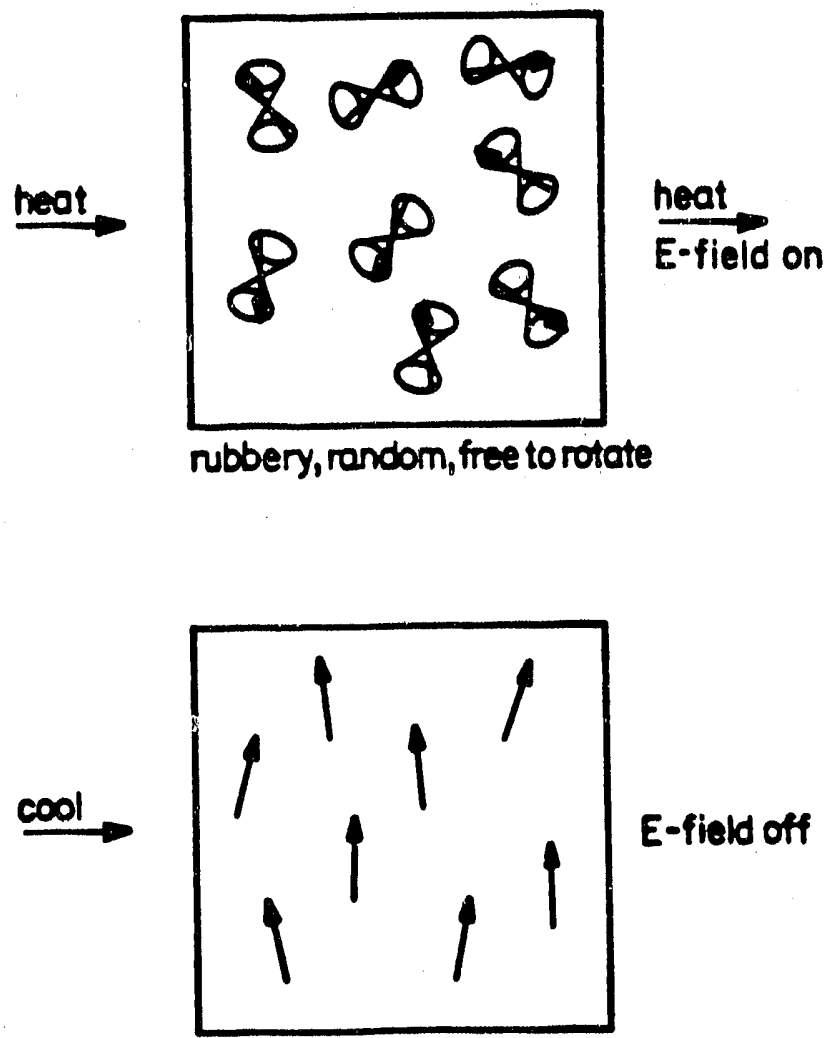

glassy, aligned

Figure 1: Poling process for thermoplastic polymer.

\section{DISCLAIMER}

Thic report was prepared as an account of work sponsored by an agency of the United States Government. Neither the United States Gnvernment nor any agency thereof, nor any of their employcis, makes any warranty, express or implied, or assumes any legal liability or responsibility for the accuracy, completeness, or usefulness of any information, apparatus, product, or process disclosed, or represents that its use would not infringe privately owned rights. Reference herein to any specific commercial product, process, or service by trade name, trademark, manufacturer, or otherwise does not necessarily const: atte or imply its endorsement, recommendation, or favoring by the United States Government or any agency thereof. The views and opinions of authors expressed herein do not necessarily state or reflect those of the United States Government or any agency thereof. 
given in Equation 1. [6] However, the electro-optic coefficient for this material is too small for electro-optic applications, and further, it was found that the susceptibility decays rather quickly in this solid solution material as shown in Figure 2. [7] Further work was carried out, both to increase the susceptibility, and to enhance the stability. A more appropriate system composed of a more optically nonlinear dicyanovinyl terminated aromatic moiety as a pendant group in a methacrylate copolymer is depicted in Figure 3.[8] The measured electro-optic coefficient $r_{33}$ was found to be $15 \times 10^{-12} \mathrm{~m} / \mathrm{V}$, which is appropriate for devices. Further, the stability in this system is greatly improved over the solid solution system as shown in Figure 2. At elevated temperatures, however, these side-chain polymers are not adequately stable. The stability can be enhanced further using a cross-linked system.

To this end, triazine polymer systems have been investigated. [9] The structure is shown in Figure 4, and includes the triazine mojety with three pendant dicyanovinyl terminated pendant nonlinear optical dyes. These polymers have been shown to possess excellent thermal stability due to the starlike network when the system is fully crosslinked. Triazines are also considered as appropriate dielectric material for electronic circuits due to their dielectric properties, stability and strength. The poling process was adapted for this thermosetting system. Films were produced of the trimer material, and then beated and poled at $150^{\circ} \mathrm{K}$ for several hours. At this temperature, the trimers polymerize into the final crosslinked network with the nonlinear optical dyes aligned in the electric field. The electro-optic coefficient, $r_{33}$, was measured to be $11 \times 10^{-12} \mathrm{~m} / \mathrm{V}$, and, again, the thermal stability of this material is again greatly enhanced with respect to the side-chain polymers as shown in Figure 2. The triazine system exhibits a high degree of stability at $85^{\circ} \mathrm{C}$. It is found, however, when applying Eq. 1 to this material, an electro-optic coefficient of approximately $20 \times 10^{-12} \mathrm{~m} / \mathrm{V}$ is expected. The reduced coefficient may be due to cis isomerization of the dye moiety, or to the presence of a potential which hinders free alignment in the presence of the electric field.

Material design considerations for electro-optic applications focus on the nonlinear optical and the polymer components both of which can be largely optimized independently. The nonlinear optical moiety design mostly involves increasing the nonlinearity while polymer design is focused on stability, optical loss, and processing. In addition, high performance devices have been fabricated and tested. [10]

\section{Second Harmonic Generation}

We have also applied multi component niaterial design to second harmonic generation materials. [11] The phase-matching condition is required for efficient second-order nonlinear optical processes. This condition occurs when the phase velocities of the various waver involved in the nonlinear optical process are equal, which is usually accomplished by adjusting the dispersion of the material. Multi component materials such as doped or functionalized polymers allow the application of anomalous dispersion in order to satisfy the phase matching condition. In this scheme, the dispersion is canceled by using the anomalous dispersion observed near an electronic excited state. For second harmonic generation of a semiconductor laser, a visible absorbing dye was selected so that the photon energy of the fundamental laser light is less than that of the dye absorption energy, and the photon energy of the second harmonic light is greater than that the dye absorption energy. In 

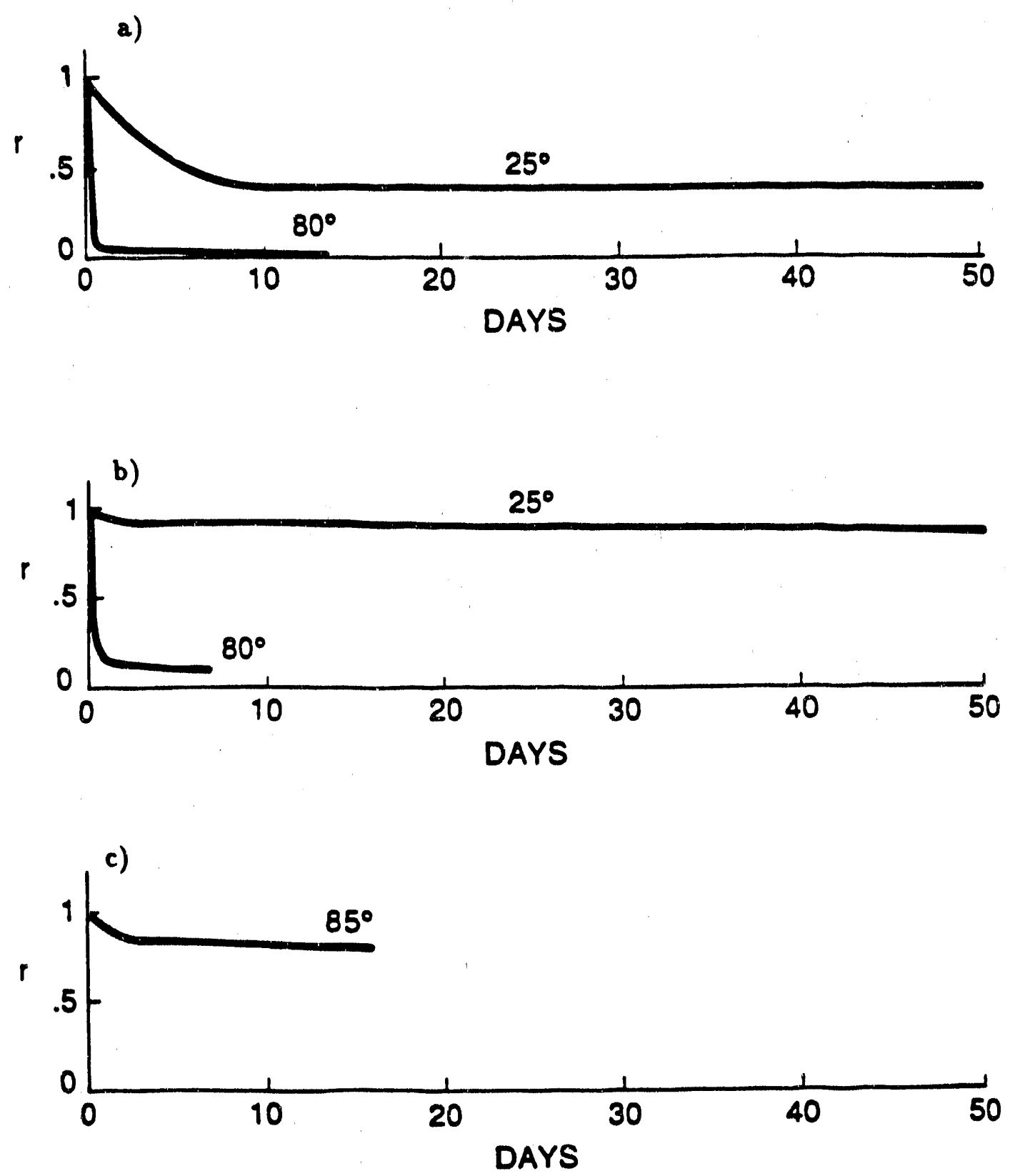

Figure 2: Decay of relative electro-optic coefficient, r. a) Disperse Red 1 in PMMA, b) DCV-MMA side-chain copolymer, c) triazine polymer. 
$6 \cdot{ }^{\circ}$

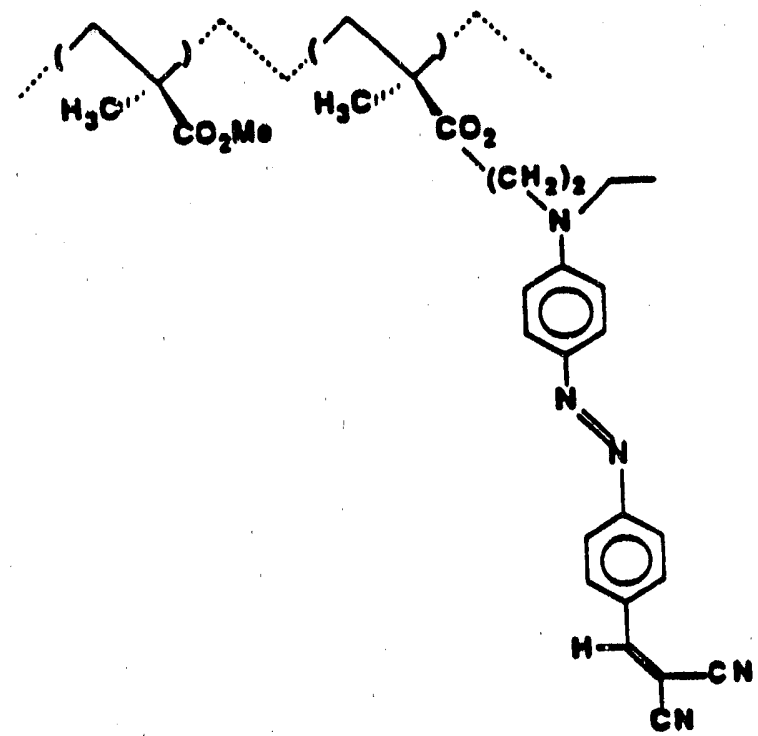

Figure 3: DCV-MMA copolymer.

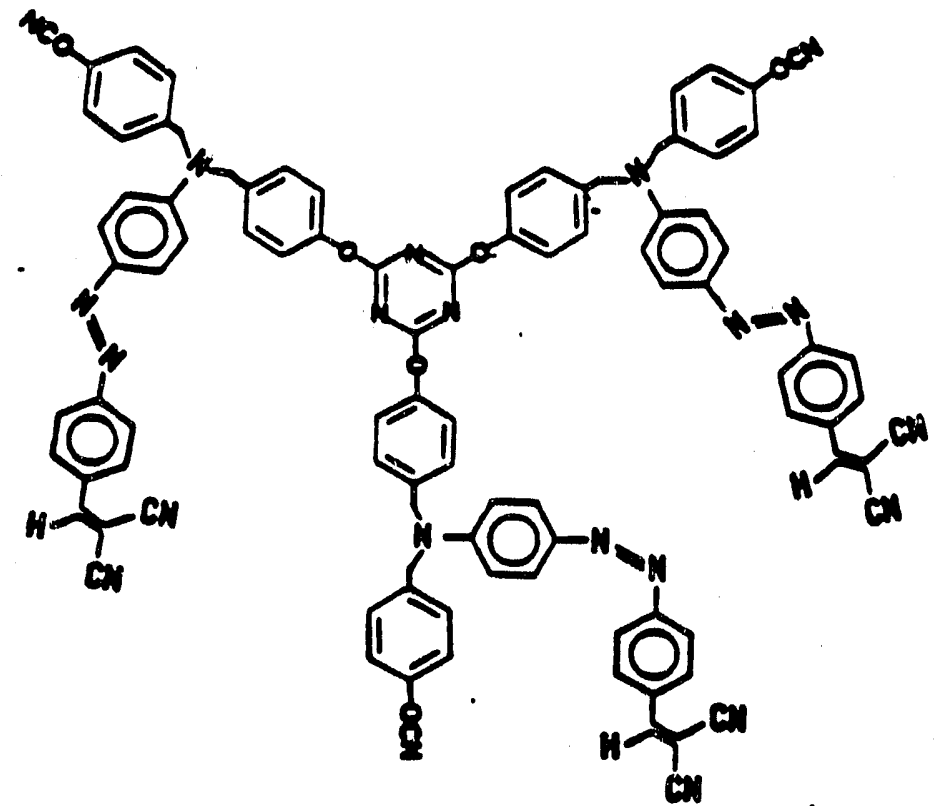

Figure 4: DCV triazine trimer. 


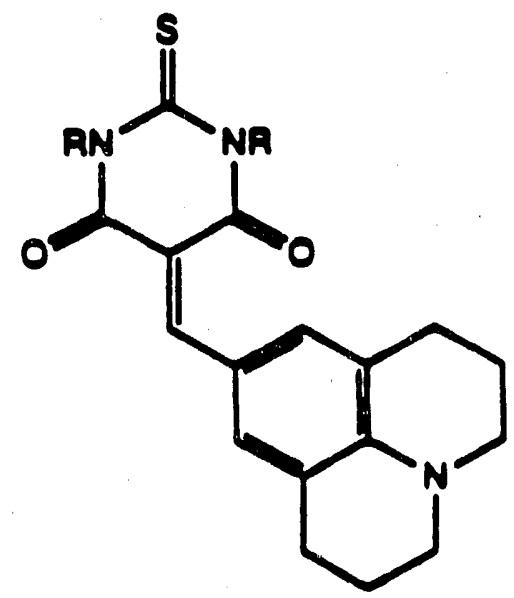

Figure 5: Barbituric acid molecule (BA).

this case, the negative molecular polarizability will reduce the polymer refractive index at the second harmonic energy due to anomalous dispersion. At a critical concentration, the phase matching condition may be achieved. [12]

This method of phase matching is attractive for several reasons. First, it allows the use of the large optical nonlinearities found in visible absorbing dyes for second harmonic generation of semiconductor laser light. This can mean a 100 to 10000 fold increase in second harmonic generation efficiency. In addition, any nonlinear optical tensor component can be utilized; especially those with their electric polarization along principle dielectric directions. In the bulk, this implies the absence of walk-off.

Phase matched second harmonic generation using anomalous dispersion was demonstrated using electric field induced second harmonic generation in liquid solution of Foron Brilliant Blue (FBB-SR) dye in acetonitrile. [12] Although the phase matched condition was attained, the conversion efficiency was limited by residual absorption of the second harmonic by the dye. A figure of merit for absorption and anomalous dispersion can be defined as the ratio of the molar absorption at the absorption peak wavelength divided by the molar absorption at the second harmonic wavelength. For FBB-SR, this figure of merit is 133. After a significant synthetic effort, it was found that the barbituric acid (BA) molecule shown in Figure 5 would be appropriate for second harmonic generation. It possesses a figure of merit of abont 540 .

Films of BA in PMMA were spin deposited onto a silicon wafers on which were grown several micron thick oxide layers. This geometry was appropriate for waveguiding purposes. The bulk indices of refraction were determined from the TE mode coupling angles. The concentration dependence of the bulk refractive index dispersion is shown in Figure 6. It is apparent that the phase matched condition is achieved at a weight fraction of about $6 \%$. The absorption coefficient in this concentration range is $20-25 \mathrm{~cm}^{-1}$. 


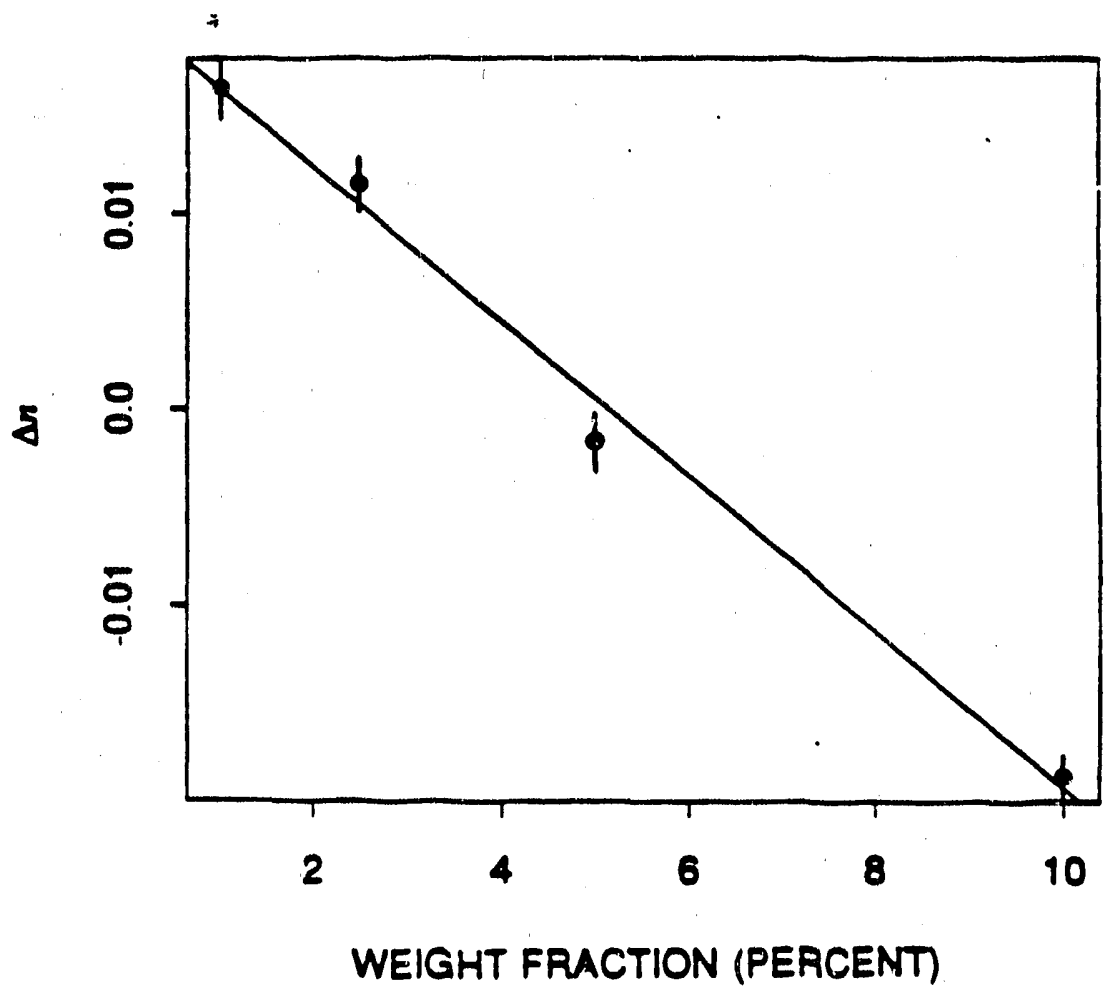

Figure 6: Dispersion of BA in PMMA versus BA concentration.

Second harmonic generation for absorbing media has been previously treated by Oudar. [13] The expected second harmonic generation efficiency, $\eta=P^{2 \omega} / P^{\omega}$ is given by,

$$
\eta=\frac{16 \pi}{c} I_{\omega} T_{Q} Q_{b}^{2} F
$$

where

$$
\begin{aligned}
T_{Q} & =2 \frac{n}{1+n} \\
Q_{b} & =\frac{32 \pi^{2} d}{\lambda(1+n) \sqrt{\left[\frac{2 \pi}{l_{c}}\right]^{2}+\alpha_{2 \omega}^{2}}} \\
F & =\exp \left[-\left(\alpha_{\omega}+\frac{\alpha_{2 \omega}}{2}\right) l\right]\left[\cosh \left(\alpha_{\omega}-\frac{\alpha_{2 \omega}}{2}\right) l-\cos \left(\frac{\pi l}{l_{c}}\right)\right]
\end{aligned}
$$

and where $l_{c}$ is the coherence length, $l$ the optical path length, $\omega$ the fundamental frequency, $\alpha_{\omega}$ the absorption at frequency $\omega, d$ the second harmonic coefficient, and $n$ the refractive index. Based on the experimental results above, the second harmonic efficiency was estimated for a $2 \mu \mathrm{m}$ square waveguide with a $100 \mathrm{~mW}$ incident intensity at a fundamental wavelength of $800 \mathrm{~nm}, l_{c}=0.1 \mathrm{~cm}, \alpha_{\omega}=0 \mathrm{~cm}^{-1}, \alpha_{2 \omega}=20 \mathrm{~cm}^{-1}, d=10^{-8} \mathrm{esu}$, and $\boldsymbol{n}=1.5$. The results as a function of optical path length, $l$, are shown in Figure 7. It is clear that even with a $0.1 \mathrm{~cm}$ coherence length, useful conversion efficiencies can be attained. It should be possible to obtain more efficient conversion by shifting to higher zero dispersion concentrations. 


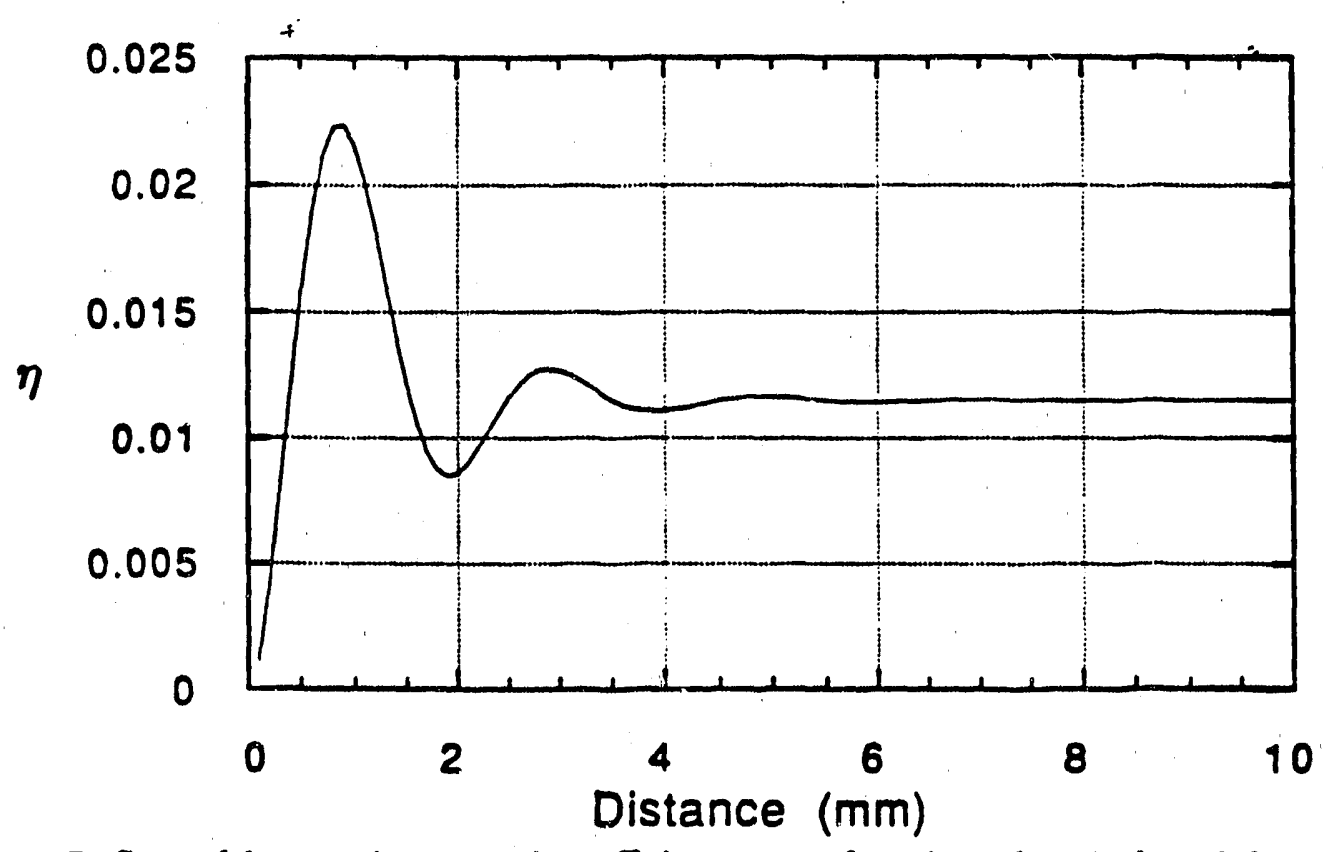

Figure 7: Second harmonic generation efficiency as a function of optical path length.

Material design considerations for anomalous dispersion phase matching also center on the nonlinear optical mojety and the polymer host. For the nonlinear moiety, the susceptibility and the optical loss spectrum must be considered, while for the polymer, the optical quality, stability, and dispersion are of prime importance in design.

\section{Controlling The Tensor Properties of the Second Order Susceptibility}

In this section, we describe how the second-order nonlinear-optical susceptibility tensor, $\chi^{(2)}$, of a dye-doped polymer can be controlled with uniaxial stress. The dopant molecules are assumed one-dimensional, where all the linear and nonlinear-optical properties arise from electronic excitations along the long axis of the molecule, which we refer to as the $z$-direction. Furthermore, it is assumed that the bulk material has axial symmetry and the molecular axis is azimuthally symmetric.

The dopants are oriented while the polymer host is elevated above its glass transicus. temperature. We assume that the orientational order is frozen in place as the material is cooled below the glass transition temperature and that the order persists over long times relative to the duration of the nonlirear-optical experiments.

In the preparation of an electric-field poled polymer, the molecules are aligned by the field above the glass transition temperature of the guest-host system. The orientational order of the dopants are determined by considering the competition between the electricfield applied torque and thermal disordering and is given by the orientational disiribution function, $G(\theta)$, as determined from the Gibbs distribution,

$$
G(\theta)=\frac{\exp \left[-\frac{\mu^{*} E}{k T} \cos \theta\right]}{\int_{-1}^{+1} d \Omega \exp \left[-\frac{\mu^{*} E}{k T} \cos \theta\right]}
$$


where $\mu^{*}$ is the dressed dipole moment of the dye molecule, $\mathbf{E}$ the magnitude of the poling field, $\mathrm{kT}$ the Boltzmann factor, $\Omega$ the solid angle, and $\theta$, the angle between the applied electric field and the dipole moment of the molecule.

Under the assumption of axial and azimuthal symmetry, the two independent tensor components of the second order susceptibility for the doped polymer are:

$$
\chi_{333}^{(2)}=N \beta_{2 z z}^{*}\left(\frac{3}{5} A_{1}+\frac{2}{5} A_{3}\right)
$$

and

$$
\chi_{113}^{(2)}=N \beta_{z 2 z}^{*}\left(\frac{1}{5} A_{1}-\frac{1}{5} A_{3}\right),
$$

where $N$ is the number density of dopants, $\beta_{z x z}^{*}$ the hyperpolarizability of the dopant, and $A_{1}$ and $A_{3}$ the order parameters of the dopant distribution as given by:

$$
A_{i}=\int_{-1}^{+1} d \Omega G(\theta) P_{i}(\cos \theta)
$$

with $P_{i}$ the $i^{\text {th }}$ Legendre polynomial.

Using the Gibbs distribution for the poled polymer, we find that the order parameters are:

$$
A_{1}=\frac{\mu^{*} E}{3 k T}
$$

and

$$
A_{3}=0 \text {. }
$$

It is clear that for these order parameters the ratio of the bulk susceptibility tensor components remains independent of the poling field and is given by:

$$
a=\frac{\chi_{113}^{(2)}}{\chi_{333}^{(2)}}=\frac{1}{3}
$$

To affect a change in this ratio, then, a torque must be applied that changes the order parameter $A_{3}$. As we show below, a uniaxial stress affects such a change.

If the thin film is stressed above the glass transition temperature along the normal to its plane, the polymer will flow in the plane radially from the stress point. Such a flow results in a torque that orients the molecules with long axis in the film plane. With both electric field and stress applied in the film normal direction, the molecules will tend to orient in ala intermediate direction between the electric field and the film plane. The stress potential, then, is minimum for $\theta=\pi / 2$ and is best approximated by the lowest order axial potential. [14] The potential is:

$$
U=b P_{2}(\cos \theta)-\mu^{*} E P_{1}(\cos \theta),
$$

where $P_{1}$ and $P_{2}$ are Legendre Polynomials. The second term is the electric-field contribution to the potential while the first term describes the axial stress potential. The coefficient $b$ describes the strength of this potential and is related to the polymer flow rate.

Under the stressed poling condition, the molecules align with the poling field, and tilt away from the plane-normal due to the polymer flow. When the sample is cooled below 


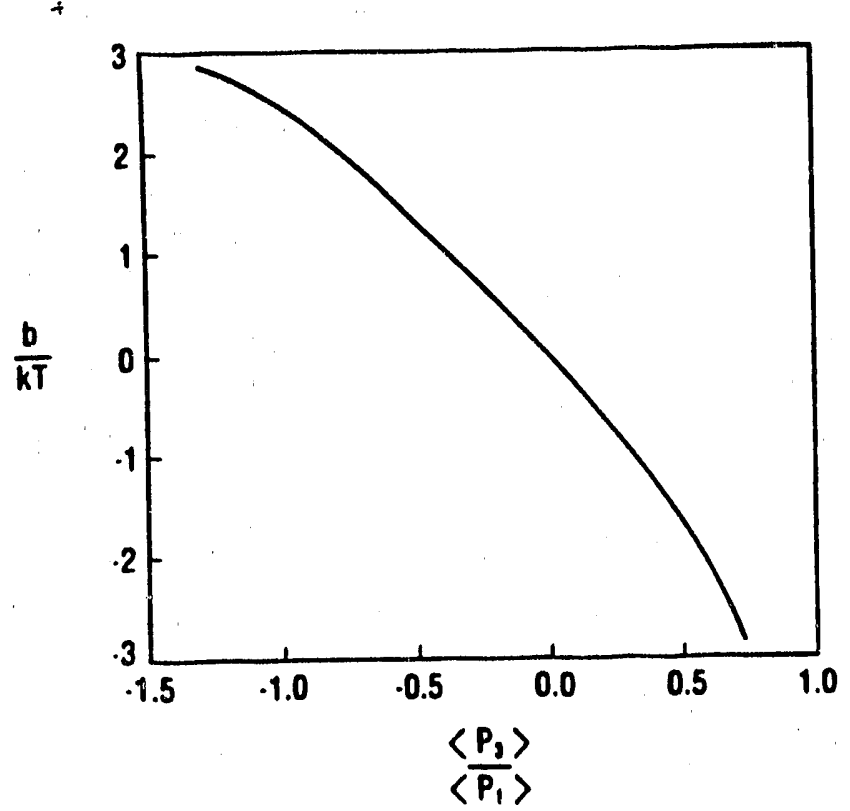

Figure 8: The calculated dependence of the order parameter ratio, $\left\langle P_{3}\right\rangle /\left\langle P_{1}\right\rangle$, on the strength of the stress potential.

the glass transition temperature, the flow stops and the molecular order is locked in. The resultant orientational order can be described by the order parameters $A_{1}$ through $A_{4}$. The stress potential is responsible for imparting axial order and results in a nonzero value of $A_{2}$ and $A_{4}$. The polar aligning electric field imparts an orientational order as described by $A_{1}$. The presence of the axial order, however, influences the effects of the poling field thereby inducing an $A_{3}$ order parameter. It is from this order parameter that the ratio of the tensor components can be controlled.

Details of this calculation are described in the literature. [14] Figure 8 shows the dependence of the order parameter satio on the degree of polymer flow, $b$. Note that for zero flow, the order parameter $A_{3}$ vanishes leaving the poled polymer results. Figure 9 shows the calculated orientational distribution functions as a function of poling field and stress. It is clear that the tensor zatio can be controlled from $a=1 / 3 \rightarrow \infty$. This range includes the ratio $a=1$, which implies an isotropic second order susceptibility tensor. Such a regime is important in the design of polarization independent optical devices.

In our experimental determination of $a$, we have found that the tensor ratio varies from $a=1 / 3 \rightarrow 0.7$ for stresses that range from $T=0 \rightarrow 3.7 \times 10^{7}$ dyne $/ \mathrm{cm}^{2}$. With increased stress levels, it may be possible to reach the polarization independent limit of $a=1$. Figure reffig:lclb summarizes the order parameters of several liquid crystals and Langmuir Blodgett films. The shaded region corresponds to that attainable through stress-poling: a new region of material orientational order.

\section{Third Order Materials}

The third order susceptibility of a material can be used for all-optical device applications. An efficient device requires both a large third order susceptibility and low loss. [15] The 
$12 \div \quad \therefore$

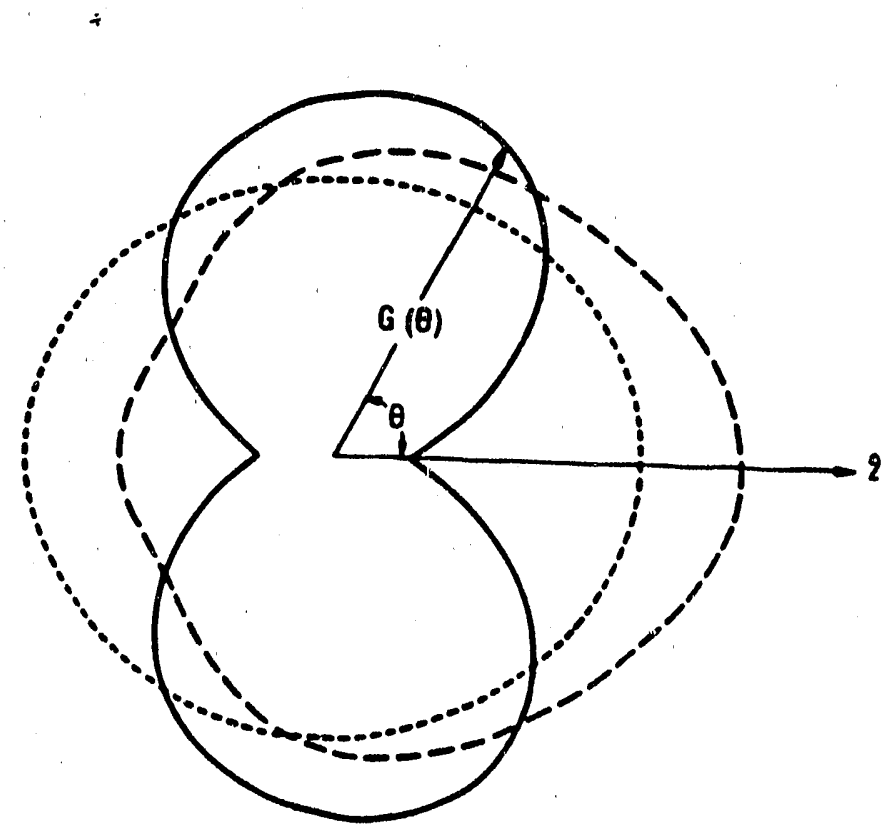

Figure 9: The orientational distribution function for a range of stresses and poling fields.

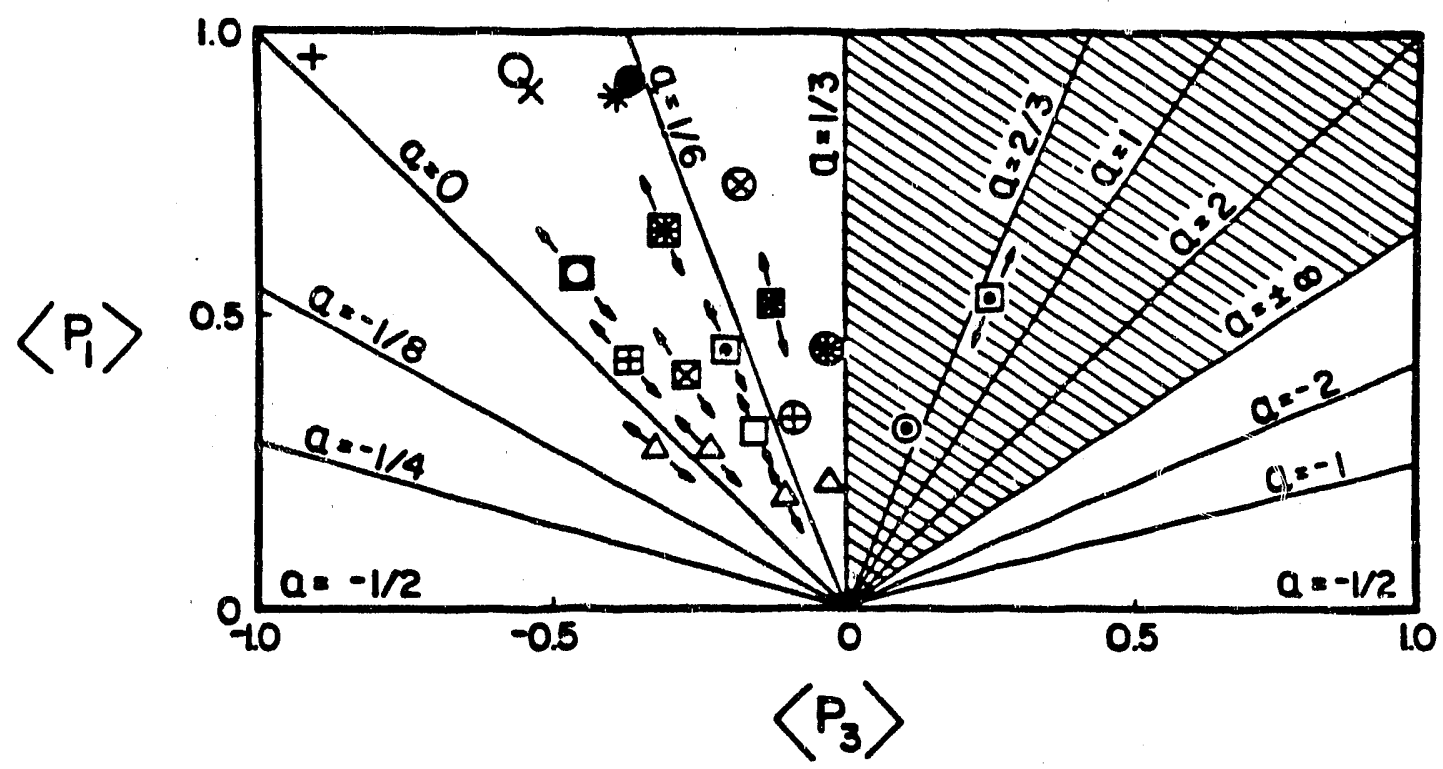

Figure 10: A survey of order parameters of Langmuir-Blodgett films and liquid crvstals. The shaded region corresponds to those orientational order parameters that are theoretically achievable through stress poling. 


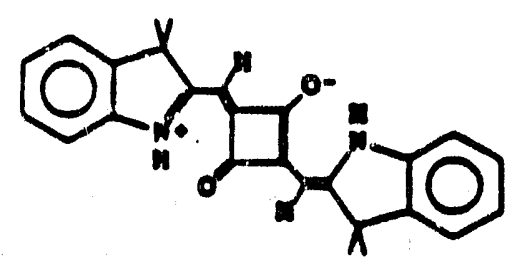

Figure 11: Squarylium dye ISQ molecalar structure.

figure of merit that best describes this material dosign consideration is the ronlinearity-loss ratio, $n_{2} / \alpha$, where $\alpha$ is the loss and $n_{2}$ defined through:

$$
\Delta n=n_{2} P \text {, }
$$

where $\Delta n$ is the intensity dependent refractive index and $P$ the power density of the applied optical beam.

In doped polymers, the bulk third order susceptibility tensor, $\chi^{(3)}$ is related to the dopant's susceptibility tensor, $\gamma:[16]$ [17]

$$
\chi^{(3)}=\Lambda<\gamma^{*}>,
$$

where $\mathrm{N}$ is the number density of dopants, $\boldsymbol{\gamma}^{*}$ the dressed third order susceptibility of the dopant and where the brackets $<>$ represent an orientational averige over the dopants distribution. Two means for optimizing the susceptibility are 1) increasing the dopant density, and 2) designing molecules with large molecular susceptibilities.

In an effort to identify large- $\chi^{(3)}$ materials, a theoretical perturbation treatment is applied to the two and three-level model of the molecular third order susceptibility. [18] In these studies, it is found that the centro symmetric two-level molecule has the largest third order molecular susceptibility if the oscillator strength to the first excited state is large. V'bile many organic molecules require more than two levels to accurately describe the observed nonlinear-optical response, [19] [20] [21] it is interesting to see where the two-level model applies, and how it may be used as a guide for material development.

Quadratic electro-optic measurements of a series of molecules shows that the centro symmetric squarylium dye ISQ as shown in Figure 11 has one of the largest third order susceptibilities. [18] This molecule has a strong and sharp transition at $650 \mathrm{~nm}$ with no other dominant states observed in the linear absorption spectrum. [22] While this suggests a two-level system, there is still the possibility that a dominant two-photon states remains undetected by virtue of its forbiclden transitions to the ground state. Quadratic electro-optic spectroscopy studies of the ISQ dye suggests that, while it may not be a purely two-level 
system, the dispersion in the third order susceptibility qualitatively approximates a twolevel response. [18] A systematic search of centro symmetric two-level systems such as the squarylium dyes may uncover systems with greatly enhanced third order susceptibilities.

Another path to improving the bulk response is to increase the dopant concentration. It has been shown that depant concentration can be substantially increased by covalently attaching the nonlinear-optical chromophores to the polymer backbone. [7] In the case of the ISQ dye, whose solubility allows doping levels of only $1 \%$ by weight, it is possible that by covalently bonding the chromophore to the polymer matrix, that number densities may be increased by an order of magnitude over the dissolved-in materials. This increase, along with future increases in the chromophore's third order susceptibility through molecular engineering, makes doped polyzne:s attractive as large $n_{2}$ materials.

The losses in the polymer system are mostly due to vibrational overtones in the near IR. At $\lambda=500 \mathrm{~nm}$, for example, losses in poly' (methyl methacrylate) (PMMA) are about $10 \mathrm{~dB} / \mathrm{km}$. [23] At more reasonable wavelengths of application, however, losses are around $1000 \mathrm{~dB} / \mathrm{km}$. Deuterating the polymers can effectively lower losses by an order of magnitude. [24] With these levels of loss, doped polymers with squarylium dyes can achieve figures of merit that exceed those of silica optical fibers.

\section{Conclusions}

Molecularly functionalized polymers can be formed into both second-and third-order nonlinear optical materials. These systems allow the separate optimization of nonlinear optical and other properties which can lead to the formation of more useful materials. Thus, waveguided electro-optic devices have be constructed out of polymeric materials which have led to the first active polymeric nonlinear optical devices. The principles of separate optimization for processability and linear and nonlinear optical properties are being also being applied to third-order nonlinear optical materials as well as second harmonic generation processes. The concept of multi component materia's will surely be applied further which will lead to new functional and multi functional materials.

\section{Referenced}

[1] A.M. Glass, Mat. Res. Bull. 13, 16 (1988).

[2] A.J. Heeger, J. Orenstein, and D.R. Ulrich, eds., Nonlinear Optical Properties c, Polymers, vol. 109 (Materials Research Society, Pittsburgh, 1988).

[3] R.D. Small, K.D. Singer, S.J. Lalama, and M.G. Kuzyk, Proc. SPIE 682, 160 (1986).

[4] K.D. Singer, J.E. Sohn, L.A. King, H.M. Gordon, H.E. Katz, and C.W. Dirk, J. Opt. Soc. Am. R 6, 1339 (1989) and references therein.

[5] K.D. Singer, J.E. Sohn, S.J. Lalami, M.G. Kuzyk, and R.D. Small, Proc. SPIE 704, 240 (1986).

[6] K.D. Singer, J.E. Sohn, and S.J. Lalama, Appl. Phys. Lett. 49, 258 (1986). 
[7] K. D. Singer, M. G. Kuzyk, W. R. Holland, J. E. Sohn, S. J. Lalama, R. B. Comizzoli, H. E. Katz, and M. L. Schilling, Appl. Phys. Lett. B3, 1800 (1988).

[8] M.L. Schilling, H.E. Kust, D.J. Cox, J. Org. Chem. 83, 5538 (1988).

[9] T. Fang (to be published).

[10] G.F. Lipscomb, R.S. Lytel, A.J. Ticknor, T.E. Van Eck, S.L. Kwiatkowski, and D.G. Girton, Proc. SPIE, 1337 (1990).

[11] P.A. Cahill and K.D. Singer, $A C S$ Symp. Ser. (in press).

!!2] P.A. Cahill, K.D. Singer, and L.A. King, Opt. Lett. 14, 1137 (1989).

[13] J.L. Oudar, J. Chem. Phys. 67, 446 (1977).

[14] M. G. Kuzyk, K. D. Singer, H. E. Zahn, and L. A. King, J. Opt. Soc. Am. B 6, 742 (1989).

[15] "Nonlinear Optical Effects in Organic Polymers," NATO ASI Series, Series E: Applied Sciences - vol 162, J. Messier, F. Kajzar, P. Prasad, and D. Ulrich, eds., Kluwer Academic Publishers, Dordrecht, 1989.

[16] M. G. Kuzyk and C. W. Dirk, Appl. Phys. Lett 54, 1628 (1989).

[17] M. G. Kuzyk, C. W. Dirk, and J. E. Sohn, J. Opt. Soc. Am. B 5, 842 (1990).

[18] M. G. Kuzyk and C. W. Dirk, Phys. Rev. A 41, 5098 (1990).

[19] C. W. Dirk and M. G. Kuzyk, Phys. Rev. A 39, 1219 (1989).

[20] J. R. Heflin, K. Y. Wong, O. Zamani-Khamiri, and A. F. Garito, Phys. Rev. B 38, 1573 (1988).

[21] Z. G. Soos and S. Ramasesha, J. Chem. Phys. 00, 1067 (1989)

[22] C. W. Dirk and M. G. Kuzyk, Chem. of Materials 2, 5 (1990).

[23] T. Kaino, Jap. J. of Appl. Phys. 24, 1661 (1985).

[24] T. Kaino, K. Jinguji, and S. Nara, Appl. Phys. Lett. 42, 567 (1983). 

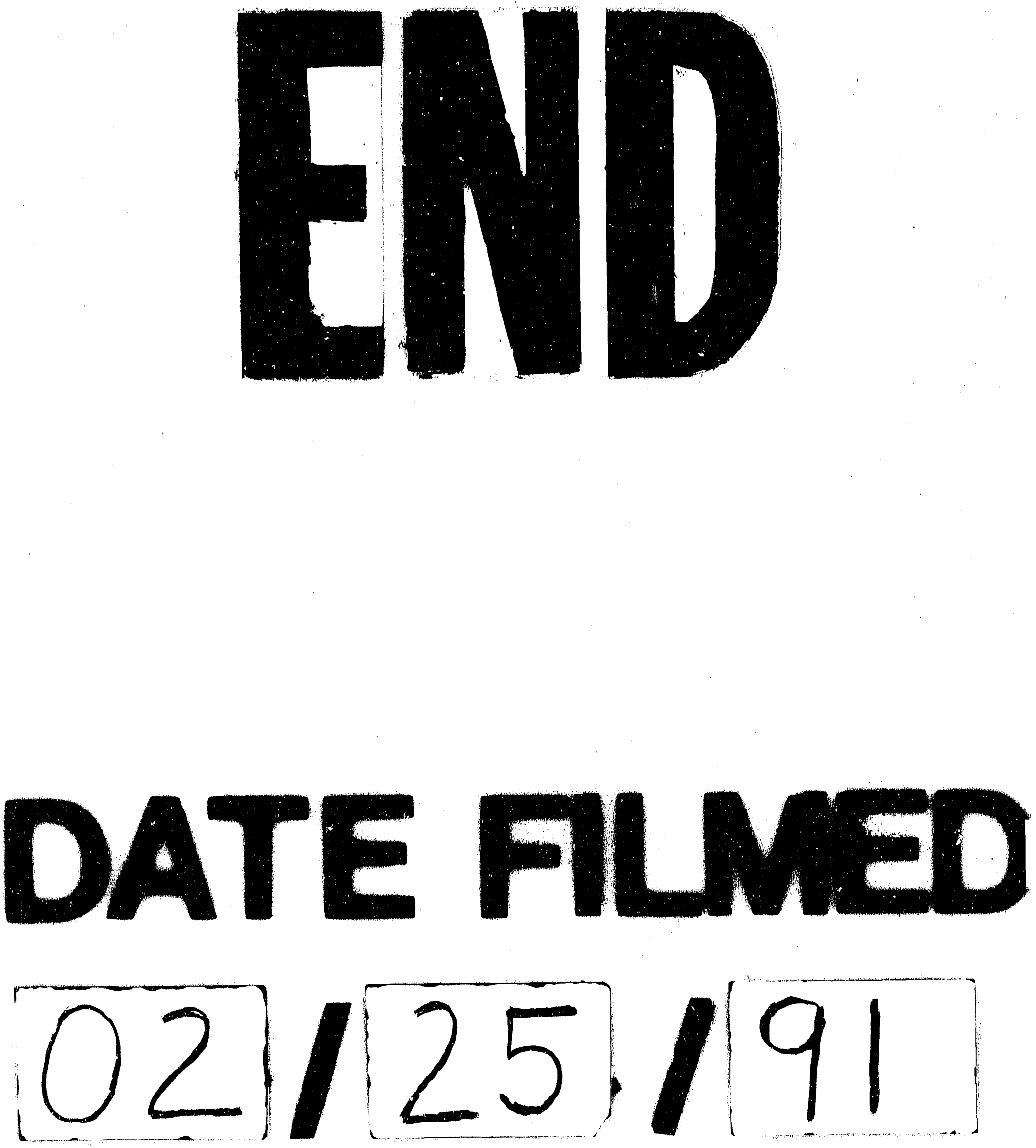
